\title{
Why do we need Tokenomics?
}

Stylianos Kampakis

UCL Centre for Blockchain Technologies, UK

Correspondence: stylianos.kampakis@gmail.com

Received: 28 April 2018 Accepted: 1 May 2018 Published: 2 May 2018

\begin{abstract}
Initial Coin Offerings (ICOs) have revolutionised the way startups are funded. Furthermore, besides their use in an ICO, tokens unlock many possibilities within the context of the artificial economies that are being set up through blockchain: from incentivisation to automatic balancing of the economy. In spite of these developments, there is still no commonly accepted theoretical framework for analysing token economies and ICOs. This presents a unique challenge to the scientific community.
\end{abstract}

Keywords: ico, initial coin offerings, ICOs, token economy, tokenomics

\section{A different landscape}

A long time has passed since 1776, when Adam Smith defined political economy in his monumental work "The Wealth of Nations". Since then economics as a scientific discipline has gone a long way with different schools of thought having shaped policy, public administration and society. However, up until now, there was one common assumptions behind all schools of thought: money is the default medium of exchange for goods and services.

However, Adam Smith, John Maynard Keynes and Milton Friedmand would find themselves in a completely different world now. Blockchain technology has allowed the development of the Initial Coin Offering and as of writing there is more than $\$ 5.6$ billion that has been raised by ICOs1. Each start-up that runs an ICO is unique. However, there is one thing common between all ICOs. This is the use of a token as a currency.

There are three different kinds of tokens:
1) Equity tokens
2) Security tokens
3) Utility tokens

Equity tokens and security tokens are simply an extension of the concept of share or asset-ownership to the blockchain.
However, it is utility tokens that offer a completely new proposition.

Utility tokens are tokens that are simply redeemable within a start-up's closed economy and are exchanged for goods and services. And this is where we observe the first major difference to what economics as a discipline has dealt with since its inception.

\section{Some examples}

The use of blockchain-based tokens allows the creation of new kinds of economies, completely customisable and adaptive, while at the same time ensuring security and transparency without a central authority. There are many possibilities, and in this article, we are going to see three different examples:

1) Improved incentivisation schemes for different agents of an ecosystem.

2) Automatic control of inflation.

3) Automatic reward/punishment of different actions within the ecosystem

Some use cases are going to be presented here.

\subsection{Improved incentivisation schemes}

Incentivising people to do something is never an easy task. In traditional economics, the assumption was that people act as rational economic agents. However, in the last few years 
economics have been greatly influenced by psychology and the work of people like the noble laureates Daniel Kahneman and Richard Thaler.

The work of Daniel Kahneman and Amos Tversky convinced us that humans are not always making decisions in a rational manner through their work in prospect theory 2. Their work demonstrated that in decisions that involve probabilities and uncertainty (which is the case for many real-world scenarios), humans tend to operate in a different way to what they would have done if they were rational agents.

Richard Thaler 3 extended some of the conclusions of this work to public policy making. In his popular book "Nudge", he explained how a government could use the cognitive biases that humans are using in decision making, in order to improve societal and economic outcomes.

Behavioural economics is a fascinating field, but the possibilities automatically increase when it is seen through the lens of blockchain technology. Through the use of tokens, it becomes possible to incentivise different agents through the production of tokens for particular actions.

For example, a user can be incentivised to purchase a product, or perform an action such as recycling. A company can be incentivised for compliance. Users can be incentivised for forming collective action. The possibilities are endless.

\subsection{Automatic balancing of an economy}

Economies go through cycles where inflation, deflation and other economic indicators fluctuate. Central banks have an indirect control over the economy through the use of interest rates, bonds and other means.

Token economies of a certain scale are not much different. However, there are two main advantages that blockchain offers:

1) Detailed data over transactions.

2) Automatic controls through the use of smart-contracts

What this means is that measures such as inflation can automatically be calculated on the spot. Safeguards against the economy, such as against speculative attacks or rapidly rising inflation, can be placed through smart contracts. Furthermore, because these measures are enforced through smart contracts, decision making is automated, transparent and available to the public. This can make token economies more robust, while also improving trust.

Indeed, a very interesting question is what would happen if central banks and governments were able to integrate blockchain within their current monetary systems.

\section{So, why tokenomics?}

So, to answer the original question posed in the beginning of the article: "why we need tokenomics"? It has become common knowledge that blockchain opens up many different possibilities. However, while tokens are a huge part of it, tokenomics is still a discipline that has not been studied in depth. A google scholar search for "token economy blockchain" returns only 1860 results.

Furthermore, the majority of ICO white papers are not using any kind of formal analysis of their economic model. For example, a model that has showed up in many ICOs, is the issuance of a limited number of tokens, which are gradually burnt as they are being used. This limits supply over time, which (if the demand is not reduced) will increase the price of the token.

While this might sound lucrative to the short-term investor, it does not explain how the model is sustainable in the long run. Indeed, ICOs is a new invention, so we don't have any examples of start-ups that followed this model and had sustainable growth for $5+$ years.

However, a proper theory of token economies would allow us to understand which models work best and are sustainable. This would also help improve the credibility of ICOs as a method of raising money, calming criticism and concerns about speculative bubbles.

All this makes it clear that the vast possibilities opened up by formalising token economies is still unexplored. The science of economics has given us powerful insights into how the laws of real world economies work. What we, as the scientific blockchain community, need to do, is spend more time and resources in understanding how to transfer learnings from traditional economics into token economies and how to create new economic models and theories that exploit the possibilities offered by blockchain.

\section{References}

[1] http://uk.businessinsider.com/how-much-raised-icos2017-tokendata-2017-2018-1

[2] Tversky, Amos; Kahneman, Daniel (1992). "Advances in prospect theory: Cumulative representation of uncertainty". Journal of Risk and Uncertainty. 5 (4): 297-323. doi:10.1007/BF00122574. ISSN 0895-5646

[3] Thaler, Richard H., and Cass R. Sunstein. Nudge: Improving decisions about health, wealth, and happiness. New Haven, CT Yales University Press, 1999. 